Mots. Les langages du politique

Sémantique et Histoire. España et Españas dans le discours de Juan Carlos

Semantics and History. España and Españas in Juan Carlos's speeches

Semántica y Historia. España y Españas en los discursos del rey Juan Carlos

\title{
Carmen Pineira-Tresmontant
}

\section{OpenEdition}

Journals

Édition électronique

URL : https://journals.openedition.org/mots/3273

DOI : $10.4000 /$ mots.3273

ISSN : 1960-6001

Éditeur

ENS Éditions

Édition imprimée

Date de publication : 1 juillet 2004

Pagination : 61-73

ISBN : 2-84788-057-7

ISSN : 0243-6450

\section{Référence électronique}

Carmen Pineira-Tresmontant, « Sémantique et Histoire. España et Españas dans le discours de Juan Carlos », Mots. Les langages du politique [En ligne], 75 | 2004, mis en ligne le 22 avril 2008, consulté le 23 avril 2022. URL : http://journals.openedition.org/mots/3273 ; DOI : https://doi.org/10.4000/mots. 3273 


\section{Sémantique et Histoire. España et Españas dans le discours de Juan Carlos}

Dans les discours annuels que le roi d'Espagne Juan Carlos a coutume de prononcer à l'occasion de Noël et quelques jours plus tard, le 6 janvier, pour la Pascua Militar, la forme España est extrêmement utilisée. Il n'y a rien d'étonnant à cela a priori. Pourtant, face à ce référent national, on dénombre trois occurrences de la forme au pluriel Españas. Quelle interprétation peut-on donner de cette coexistence surprenante et, plus largement, de l'usage du nom de l'Espagne dans le discours du roi?

\section{Discours de roi}

Installé sur le trône par les lois de succession imposées par Franco, Juan Carlos ouvre, dès son avènement en 1975, une «transition démocratique » qui aboutira trois ans plus tard à l'adoption d'une Constitution parlementaire. Par la suite, il défendra la jeune démocratie espagnole contre les tentatives de retour à l'ancien régime franquiste. Il favorisera ensuite l'alternance politique en désignant le socialiste Felipe González comme Premier ministre. Cette ouverture démocratique sera à l'origine d'un processus d'intégration de l'Espagne dans l'Union européenne. En Espagne, le monarque ne gouverne pas; le pouvoir exécutif est aux mains d'un gouvernement issu de la majorité parlementaire et des représentants élus des différentes régions «autonomes».

Pourtant, aux yeux de ses contemporains, c'est le roi qui a incarné la modernisation et la transformation du pays. Constitutionnellement, il est aussi le chef des armées. Dans le cadre de ses différentes fonctions, le roi s'adresse fréquemment aux Espagnols tantôt lors d'interventions ritualisées (Noël, Nouvel An, etc.), tantôt de façon plus protocolaire. Enfin, par deux fois, lors d'évène-

\footnotetext{
${ }^{1}$ Linguistique hispanique - Université Paris 8-CPineiraT@aol.com
} 
ments très graves il s'est adressé à la nation: en février $1981^{2}$ et, tout récemment, à la suite des attentats de Madrid du 11 mars 2004.

Le corpus sélectionné pour cette étude ${ }^{3}$ est constitué de deux séries de 25 discours chacune. Les premiers sont adressés par le roi à l'ensemble des Espagnols, chaque année au moment des fêtes de Noël. Ces textes sont réunis dans une première série que nous appellerons Navidad, qui va de décembre 1975 à décembre 1999. La seconde série du même type (désignée par Pascua), qui va de janvier 1976 à janvier 2000, est constituée des discours prononcés quelques jours plus tard, le 6 de ce mois, à l'occasion de la cérémonie de la Pascua Militar, au cours de laquelle le roi formule ses vœux à l'intention des membres des forces armées. Deux dates fixes, deux publics aux caractéristiques dissemblables, deux contextes de communication politique; mais un seul émetteur dans une fonction identique, presque aux mêmes dates et périodes de vœux à la jointure de deux années. L'intérêt comparatif est évident. Mais focalisons-nous ici sur les usages et contextes d'España et d'Españas.

\section{L'usage particulier des noms propres}

Il est classique de définir ainsi le nom propre ${ }^{4}$ :

Les noms propres s'écrivent avec une majuscule, n'ont pas de déterminants (Pierre, Paris) ou bien se construisent avec un déterminant contraint, l'article défini (le Rhin, les Vosges). Si, comme les noms communs, ils désignent des personnes, des objets, des lieux, etc., ils sont pourtant dépourvus de sens lexical: ils n'entretiennent pas de relations sémantiques (de synonymie, d'hyponymie ou d'antonymie). D'où le débat toujours ouvert, surtout chez les philosophes du langage, pour savoir si les noms propres ont un sens. À quoi, le grammairien peut répondre que le «sens» d'un nom propre ordinaire réside dans le mode de désignation qu'il opère.

Le nom propre (désormais $\mathrm{Np}$ ) peut être caractérisé par le fait qu'il indique une qualité appartenant à un seul individu (ou institution, ou concept). Cependant cette définition simple s'interprète traditionnellement de façon contradictoire. La première thèse pose que le $\mathrm{Np}$ constitue une marque individualisante mais vide de sens $s^{5}$ : le Np n'indique pas par lui-même, c'est-à-dire par sa graphie ou

2. Tentative de coup d'État militaire du colonel Tejero faisant irruption à la tête de ses troupes dans la salle des Cortès le 23 février 1981.

3. Le corpus d'ensemble compte 32196 occurrences pour Navidad et 32407 occurrences pour Pascua.

4. M. Riegel et al., 1998, Grammaire méthodique du français, Paris, PUF.

5. F. Récanati, 1983, «La sémantique des noms propres : remarques sur la notion de désignateurs rigides », Langue française, 1957, p. 106-118. 
par sa morphologie, de signification particulière quant aux caractéristiques de l'entité concernée. Il est asémantique. La seconde thèse soutient, à l'inverse, que le Np porte l'ensemble des référents sémantiques connus de l'entité concernée. C'est une «marque distinctive pleine $»^{6}$. Cette thèse considère que les $\mathrm{Np}$ ont une réelle valeur sémantique.

Ces conceptions opposées sont intuitivement présentes dans le langage quotidien, puisque nous pouvons interpeller Paul / Pablo sans accorder de signification particulière à ce prénom et en même temps nous nous adressons au Père Noël ou à San Pedro comme à une entité individuelle, référencée et désignée par un nom propre distinctif. Ce constat nous amène à une conclusion simple: il n'est pas possible d'identifier le champ sémantique d'un nom propre sans prendre en considération son usage contextuel et situé chez un émetteur donné. Plus précisément, le fait de désigner une réalité, matérielle, vivante ou symbolique, à l'aide d'un Np revient à le baptiser d'une appellation, c'est-à-dire à lui associer formellement un ou plusieurs référents à un moment historique déterminé. Bien entendu, cette association formelle est limitée dans le temps et dans l'espace, puisque d'autres «baptêmes » peuvent survenir et remplacer l'appellation d'origine, ou d'autres cultures peuvent choisir d'autres désignations.

Nous retrouvons cette idée appliquée à la langue espagnole ${ }^{7}$ :

El nombre propio no hace más que particularizar el individuo para que después, inevitablemente, se llene con frecuencia de significado cualificador y distintivo para un grupo restringido de hablantes. (Le nom propre ne fait que singulariser l'individu pour qu'ensuite, inévitablement, il se charge fréquemment de signification qualificative et distinctive pour un groupe restreint de locuteurs.)

Tout ceci revient à poser que l'analyse des Np est possible dans l'espace discursif, qui est par nature constitué de textes datés, identifiés, contextualisés.

\section{Rencontres d'un roi et d'un nom propre au singulier}

La forme España est utilisée 154 fois dans les discours de Navidad et 108 fois dans ceux de la Pascua. On observe une présence de cette forme tout au long de la période considérée, même si le roi l'utilise inégalement selon les années. La première remarque sur cette utilisation est qu'España peut apparaitre sans déterminant, comme une entité disposant d'une personnalité identifiée. Voici quelques exemples pris dans l'une et l'autre séries textuelles:

6. G.-E. Serfati, 2000, «Le statut lexicographique du nom propre: remarques méthodologiques et linguistiques», Mots, $\mathrm{n}^{\circ}$ 63, p. 106.

7. J. A. Franch et J. .M. Blecua, 1994, Grammatica española, Barcelone, Ariel, p. 502. 
Navidad:

- nos unamos para que España marche hacia (unissons-nous pour qu'Espagne aille de l'avant);

- también ha ido bien España (Espagne s'est également bien portée);

- un proyecto de vida en común que se llama España (un projet de vie en commun qui s'appelle Espagne).

Pascua :

- nosotros que consagramos nuestra vida a España (nous autres qui consacrons notre vie à Espagne);

- para mantener la unidad de España (pour maintenir l'unité d'Espagne);

- «viva España!» (vive Espagne!);

- España sabe que permanecéis en vela permanente (Espagne sait que vous êtes sur vos gardes en permanence).

Dans ce type d'emploi, le roi donne à España, personnifiée, un rôle protecteur, quasi maternel. Il évoque l'idée d'une fusion dans un projet de «vie en commun» et, s'agissant des militaires, du sacrifice envers la mère patrie.

De façon complémentaire, les deux types de discours présentent de nombreuses attestations de la forme España employée avec une grande diversité de déterminants: articles indéfinis (una), définis (la), adjectifs possessifs (nuestra), démonstratifs (esta), indéfinis (toda)... S'agit-il, dans tous ces cas, de renforcer la réalité nationale en appuyant son caractère total, voire unique?

Navidad:

- para una España más fuerte, más libre, más estable (pour une Espagne plus forte, plus libre et plus stable);

- el esfuerzo de cada uno de vosotros para una España mejor (l'effort de chacun d'entre vous pour une Espagne meilleure);

- una España unida (une Espagne unie);

- optar entre «una España que muere y otra que bosteza» (choisir entre «une Espagne qui meurt et l'autre qui baille»);

- nuestro común anhelo de una España mejor (notre désir commun d'une Espagne meilleure);

- la España del pasado con la del futuro (l'Espagne du passé avec celle du futur) ;

- la España de los sueños (l'Espagne de nos rêves);

- nuestra España pequeña y cordial (notre Espagne petite et cordiale); 
- nuestra España es la misma que la de nuestros hijos (notre Espagne est la même que celle de nos enfants);

- nos sentimos solidarios de esta España que nos ampara (nous nous sentons solidaires de cette Espagne qui nous protège).

Pascua:

- estamos construyendo día a día una España mejor (nous construisons jour après jour une Espagne meilleure);

- el pasado, el presente y el porvenir de la España eterna (le passé, le présent et le futur de l'Espagne éternelle);

- la España de hoy no renuncia ni teme al ayer (l'Espagne d'aujourd'hui ne renonce ni ne craint celle d'hier);

- la España eterna (l'Espagne éternelle);

- una norma de conducta de la que toda España ha de extraer una lección (une ligne de conduite dont toute l'Espagne doit tirer une leçon;

- sé que toda España viví intensamente este episodio dificil (je sais que toute l'Espagne a vécu intensément ce moment difficile);

- alcanzar esa España mejor (atteindre cette Espagne meilleure).

Observons en premier lieu l'opposition entre les déterminants utilisés dans Navidad et ceux employés dans Pascua. Dans les discours de Navidad, le roi utilise la forme España accompagnée d'une connotation affective: nuestra España, mais aussi nuestro anhelo, nuestros hijos, de los sueños. Cette association confère aux discours une dimension collective, partagée entre le roi et les citoyens espagnols. Dans Pascua, la détermination définie désigne une entité placée sur l'axe du temps : la España eterna, la España de hoy. Cet usage constraste avec l'utilisation collective et affective faite dans Navidad. En revanche, la détermination indéfinie marque un positionnement flou et mal identifié du nom $^{8}$ : toda España, una España mejor. On est loin de la personnification d'España comme acteur, référent ou projet commun. Seule son unité intangible est ici fortement appuyée (toda, una, eterna): l'armée n'en est-elle pas garante? On note aussi une intéressante opposition entre «Alcanzar esa España mejor» de Pascua et «esta España que nos ampara» de Navidad: la désignation proposée aux militaires demeure distanciée, tout à fait indéfinie et, pour tout dire, rhétorique, alors que l'Espagne protectrice invoquée dans les discours à la nation entretient une relation étroite avec un nos où roi et peuple ne font qu'un.

8. C. Pineira-Tresmontant, 1988, «Rigidités discursives et flou sémantique: la notion de lexie», Mots, $\mathrm{n}^{\circ} 17$, octobre, p. 145-169. 
Les caractéristiques relevées dans les occurrences de la forme España précédée d'un déterminant ont la particularité de placer le nom soit dans la position d'une personnalité désignée et reconnue, même si elle est différente dans Pascua et dans Navidad, soit dans une position non identifiée, non reconnue et homogène dans les deux séries de discours. On a affaire à une opposition marquée qui passe par le choix de la détermination définie ou indéfinie: dans le premier cas, une signification individualisée est partagée entre l'émetteur et les récepteurs; dans le second, la formule est vide de contenu précis autre qu'une connotation glorieuse.

\section{Españas, un pluriel historiquement signifiant}

Dans notre corpus, la forme Españas peut posséder le morphème de nombre des noms communs. Elle est aussi précédée de déterminants peu habituels : indéfini (otras), numéral (dos) et défini (las). Qu'en disent les grammaires?

En règle générale le nom propre ne porte pas la marque du pluriel, étant donné son caractère d'unicité. Cependant, il peut, selon un usage assez indécis, recevoir cette marque dans les cas où il désigne plusieurs individus d'un sous-ensemble: les Dupont, mais : [...] les Goyas du Prado, [...] une opposition entre deux Frances 9 .

Los nombres propios de persona pueden tomar el plural segùn la norma de la lengua ${ }^{10}$.

Or le roi Juan Carlos utilise, dans notre corpus, trois fois la forme Españas, une fois dans les discours de Navidad (en 1978) et deux fois dans ceux de la série Pascua (en 1980 et 1982). Ces trois extraits en montrent l'intérêt:

- Así me lo han recordado, una y otra vez, en las naciones iberoamericanas que he visitado, las que forman otras Españas con las que nacemos en la misma lengua y en el mismo espíritu. Ellas nos están mirando, en esa perspectiva ejemplarizadora que ha sido siempre el norte de nuestra Historia patria. (Navidad, 1978) (C'est ce qu'on m'a rappelé parfois dans les pays ibéro-américains que j'ai visités, ceux qui forment d'autres Espagnes avec lesquelles nous partageons dès notre naissance la même langue et le même esprit. Elles nous regardent dans cette perspective d'exemplarité qui a toujours été le sens de l'Histoire de notre patrie.)

- Esta tradicional conmemoración de la Pascua Militar, iniciada en 1782 por Carlos III, Rey de las Españas, me permite reuniros de nuevo aqui, en la festividad de la adoración de los Reyes Magos. (Pascua, 1980) (Cette tradition commémorative de Pascua Militar entreprise en 1782 par Carlos III, roi des Espagnes, me permet de vous réunir de nouveau ici pour célébrer la fête de l'adoration des Rois Mages.)

9. P. Charaudeau, 1992, Grammaire du sens et de l'expression, Paris, Hachette, rééd. 1996, p. 24-25.

10. J. A. Franch et J. .M. Blecua, 1994, op. cit., p. 545. 
- No se puede olvidar y quiero decirlo claramente, aunque estemos ya muy alejados de la lucha que enfrento a dos Españas, de sus antecedentes y de sus consecuencias, que en esa guerra civil, trágica y dolorosa... (Pascua, 1982) (On ne peut oublier, je veux le dire clairement, même si nous sommes déjà très éloignés de cette lutte qui a opposé les deux Espagnes, de ses causes et de ses conséquences, que dans cette guerre civile tragique et douloureuse...)

Dans ces trois citations, le roi évoque trois réalités parfaitement distinctes et identifiées à l'aide de la variation du seul déterminant. Le déterminant indéfini otras désigne, dans le discours de Navidad, les pays issus de la Conquista (les «nations ibéro-américaines »); otras souligne qu'il existe une communauté de langue et peut-être d'esprit mais pas de «nuestra Historia patria». Le déterminant défini las, utilisé dans le discours adressé aux militaires, fait mention d'une réalité historique ancienne, précise, établie, avec des subdivisions politiques et institutionnelles placées sous l'autorité de la Couronne. Le syntagme nominal las Españas évoque ici la Hispania Romana divisée administrativement en Citerior (Intérieure) et Ulterior (Extérieure), puis la division politique de la Péninsule en plusieurs royaumes après la disparition du Regnum Hispaniae romano-visigodo, ainsi que l'institution du confédéralisme par les Rois Catholiques. Quant au déterminant numéral dos, dans 1'expression «dos Españas », il marque une division politique, sans frontières officielles. Celle-ci identifie une fracture qui sépare la société espagnole non seulement pendant la période de la guerre civile mais aussi jusqu'au moment de la «transition» conduite par le roi. Les frontières de cette fracture ont existé au cours de la guerre civile: il suffit de regarder les cartes militaires de l'avancée des troupes nationalistes contre le gouvernement de la République ${ }^{11}$; elles ont perduré dans les esprits, c'est-à-dire sans existence sur le terrain, après ce que le roi appelle, devant les militaires, la victoria.

Ces trois emplois d'Españas servent au roi, de façon implicite, d'antithèse à España, en tant que nomination incarnant une forme ancienne, provisoire, dépassée ou rejetée d'anti-Espagne. Dans cet usage, le pluriel renforce par antithèse la signification unificatrice et univoque, éternisée, du singulier España. Ainsi, le syntagme las Españas reflétait au $18^{\mathrm{e}}$ siècle l'appellation donnée à l'empire, avec la péninsule ibérique et les terres conquises d'Amérique latine. De nombreux épisodes historiques ont laissé des traces dans la mémoire espagnole, telle la guerre de Cuba au tournant du $20^{\mathrm{e}}$ siècle, avec le sentiment d'un monde perdu et du nécessaire retour vers les forces vives qui incarnent

11. On peut examiner à ce propos les cartes des lignes d'affrontement des troupes franquistes et des forces républicaines dans R. Tamame et S. Quesada, 2001, Panoroma de la formacion de Espana y de las culturas hispanicas. Imagenes de Espana, Madrid, Edelsa, Grupo Didascalias. 
l'éternelle âme espagnole. Le syntagme las otras Españas évoque les terres conquises et perdues, mais où l'Espagne maintient sa langue et des liens privilégiés. Sans être péjoratif, otras marque une irréductible différence d'avec l'Espagne européenne, implicitement la véritable Espagne, c'est-à-dire l'unique.

Toutefois l'expression la plus marquante est las dos Españas, parce que toutes les générations de citoyens espagnols, anciens comme jeunes, connaissaient en 1982 le contexte sémantique de ce syntagme ${ }^{12}$. Tel n'est peut-être plus le cas aujourd'hui, vingt ans plus tard. C'est en 1982, juste après le coup d'État avorté du colonel Tejero, que Juan Carlos évoque, dans son discours de la Pascua Militar, l'expression si connotée «las dos Españas». Il faut s'arrêter un moment sur ce point et rappeler avec précision le sens auquel cette expression fait écho dans l'esprit des auditeurs militaires de Juan Carlos.

Au cours des années trente, la España des Républicains est un «pueblo de trabajadores ${ }^{13}{ }^{13}$ (peuple de travailleurs). À l'inverse, la phalange refuse cette Espagne «que està vendida» (qui est vendue) et se fonde sur les slogans célèbres de José Antonio Primo de Rivera: «Una, grande y libre. Patria, el pan y la justicia» (Une, grande et libre. Patrie, le pain et la justice), dotée d'une «unidad de destino en lo Universal $\gg^{14}$ (unité de destin dans l'Universel). Il y avait bien là deux Espagnes. Ce concept se traduit à l'époque en zones délimitées au cours de la guerre civile, avec une vraie lutte de frontières. Max Aub, dans son livre El campo del Moro, décrit ainsi ce qu'il voit de son avion:

... despegan. Desde que toman altura, ven la línea que parte España: Fuencarral, de ellos; Vicálvaro, nuestro; Carablanchel, de ellos; Vallecas, nuestro; Villaverde, de ellos... (... ils décollent. Dès qu'ils prennent de l'altitude, ils voient la ligne qui partage l'Espagne: Fuencarral, à eux; Vicalvaro, à nous; Carablanchel, à eux; Valleca, à nous; Villaverde, à eux...) $)^{15}$

Pour la droite espagnole, la «anti España» est inadmissible: elle est «roja» (rouge), «masónica» (maçonique), «apostasia» (hérétique). À ce sujet, les affirmations sont multiples:

La anti-España está representada por judios, heresiarcas, protestantes, comuneros, moriscos, enciclopedistas, afrancesados, masones, krausistas, liberales y marxistas, orda de ladrones, asesinos, incendiarios y criminales que son la Revolución. (L'antiEspagne est représentée par les juifs, les hérésiarques, les protestants, les comune-

12. Voir en annexe les origines historiques du syntagme «les deux Espagnes».

13. J. Ortega y Gasset, 1969, Obras completas, Madrid, Revista de Occidente, tome 11, p. 138.

14. J. A. Primo de Rivera, 1945, Obras completas, Madrid, Ediciones de la Vicesecretaria de Educacion popular de FET y las JONS.

15. M. Aub, 1969, El campo del Moro, Andorre, Editorial Andorra, cité par M. A. Rebollo, 1978, Lenguage y politica, Valence, F. Torres, p. 103. 
ros, les morisques, les encyclopédistes, les francisés, les franc-maçons, les krausistes, les libéraux et les marxistes, les hordes de voleurs, d'assassins, d'incendiaires et de criminels qui représentent la Révolution.) ${ }^{16}$

En 1979, Manuel Fraga, fondateur de Alianza Popular (parti de droite), peut bien affirmer: "yo creo que si hay que escoger entre España y la democracia, es mejor escoger España ${ }^{17}$ (je crois que s'il faut choisir entre Espagne et la démocratie, il vaut mieux choisir Espagne), cela n'empêche pas qu'au même moment l'Union du centre démocratique (UCD), qui obtient la majorité aux premières élections législatives de mars 1979, exprime une rupture claire avec cette conception encore teintée de franquisme :

UCD expresaba su voluntad de romper el trágico dilema de las dos Españas, que ha marcado nuestra historia durante los dos últimos siglos. (Proposición electoral, UCD, 1979) (L'UCD exprime sa volonté de rompre le tragique dilemme des deux Espagnes qui a marqué notre histoire pendant les deux derniers siècles.)

Terminons l'illustration des relations entre sens et histoire, où se joue le destin d'un nom propre, par cette citation de Francisco Franco qui enracine l'España de son époque dans la guerre civile: «España es una unidad forjada en los campos de batalla que ha alimentado con una obra politica y social durante treinta años ${ }^{18}$ (Espagne est une unité forgée sur les champs de bataille qu'elle a nourrie d'une œuvre politique et sociale pendant trente ans).

L'exercice mené par le roi à l'aide de changements de déterminants n'a pas pour seul but d'adapter son discours à son auditoire; bien plus profondément, il consiste à déconstruire le sens traditionnel assumé par la forme plurielle Españas qui est un héritage historique, déjà ancien, celui qui est associé à la figure de Carlos III (voir discours de la Pascua Militar de 1982). Il ajoute à cette signification deux autres acceptions originales et fait, en ce sens, œuvre créatrice. Surtout, ces trois acceptions mises côte à côte lui permettent de reléguer tout ce qui n'est pas le cœur indissoluble de l'Espagne qu'il propose pour le futur. À cet égard, il est important que les acceptions qu'il ne valide pas en tant que roi soient marquées par la forme du pluriel et donc opposées morphologiquement à la forme au singulier, c'est-à-dire unitaire, qu'il cherche à promouvoir au-delà des divisions passées et des oppositions internes. Notons enfin que

16. Cité par J. de Santiago Guervos, 1992, El léxico politico de la transicion politica, Universidad de Salamanca, p. 197.

17. M. Fraga, Cambio 16, 14-20 octobre 1979.

18. F. Franco, 1971, Discursos y mensajes del Jefe del Estado, 1968-1970, Madrid, Publicationes españolas, p. 21. 
le roi n'utilise plus cette forme au pluriel depuis janvier 1982. À partir de cette date, España est perçue comme unique non seulement en tant que référent incontesté mais également par son mode de désignation au singulier. Dans son discours du 12 mars 2004, le roi fait appel à l'Espagne comme à une personne unique, douée de cœur et de sens moral:

La barbarie terrorista ha sumido hoy a España en el más profundo dolor, repulsa e indignación. [...] La España de buen corazón ha vibrado una vez más ofreciendo su ayuda material y apoyo moral. [...] El terrorismo nunca conseguirá sus objetivos. No conseguirá doblegar nuestra fe en la democracia, ni nuestra confianza en el futuro de España. (La barbarie terroriste a plongé aujourd'hui Espagne dans la douleur la plus profonde et suscité condamnation et indignation. [...] L'Espagne au grand cœur a vibré une fois de plus en offrant aide matérielle et appui moral. [...] Le terrorisme n'atteindra jamais ses objectifs. Il ne parviendra pas à ébranler notre foi en la démocratie, ni notre confiance en l'avenir d'Espagne.) 


\begin{abstract}
Annexe
D'où vient le syntagme «las dos Españas»? Un peu d'histoire ${ }^{19}$ : l'expression «las dos Españas» apparait dès le $18^{\mathrm{e}}$ siècle, lorsque les idées françaises de liberté et de modernisme suscitent en Espagne un mouvement réformiste sous la monarchie des Bourbons. L'irruption des idées nouvelles a divisé les élites espagnoles entre partisans de l'étranger et défenseurs de la tradition nationale ou «casticistas». Ce dernier qualificatif évoque aussi bien la pureté que le raffinement linguistique ou le respect des usages traditionnels. Les $18^{\mathrm{e}}$ et $19^{\mathrm{e}}$ siècles voient un mouvement de gens, «los afrancesados», fascinés par la langue, et qui créent «el afrancesamiento ». Rappelons ici que Napoléon installe au pouvoir son frère Joseph en prenant appui sur les «afrancesados». Cette opposition idéologique et politique est à l'origine de la scission en deux Espagnes qui a perduré jusqu'à nos jours.

Malgré la réaction conservatrice appuyée sur le patriotisme anti-étranger, l'Espagne réussit à s'ouvrir progressivement à l'esprit des Lumières et à se développer économiquement. Cette modernisation s'est cependant trouvée freinée par l'ascendant d'une Église conservatrice résistant à la diffusion des Lumières et alliée aux défenseurs les plus farouches de l'Ancien Régime contre la minorité moderniste et bourgeoise. Ainsi, la brèche qui s'est ouverte fut plus qu'un combat d'idées; elle a été véritablement nourrie par les luttes d'intérêts et les logiques de développement économique antagonistes qui ont parcouru les $19^{\mathrm{e}}$ et $20^{\mathrm{e}}$ siècles. Il est singulier que la même expression «las dos Españas» ait pu recouvrer cette valeur idéologique alors que le pays a profondément changé ses modes de développement économique et ses relations politiques intérieures au cours des deux siècles.

Un même syntagme a donc symbolisé les Lumières au $18^{\mathrm{e}}$ siècle, les idées révolutionnaires françaises au début du $19^{\mathrm{e}}$ puis la révolution industrielle au $20^{\mathrm{e}}$ siècle pour incarner finalement l'opposition du phalangisme aux idées républicaines au cours de la guerre civile et durant l'époque franquiste. Cet antagonisme séculaire fut dit par Antonio Machado:

Españolito que vienes

Al mundo, te guarde Dios

Una de las dos Españas

Ha de helarte el corazón.
\end{abstract}

${ }^{19}$ Outre R. Tamames et S. Quesada, 2001, mentionné plus haut, on pourra se référer à : M. A. Rebollo Torio, 1978, Lenguaje y politica, Valence, Fernando Torres. 


\section{(Jeune Español qui vient}

\section{$\mathrm{Au}$ Monde, Dieu te protège \\ L'une des deux Espagnes \\ Doit te glacer le cœur.)}

Pour Machado, durant la guerre civile, il était impossible de réunir ces visions opposées des deux Espagnes ${ }^{20}$.

Les Constitutionalistes des Cortès et du Sénat à la fin des années 1970 étaient bien conscients de l'antagonisme. Interrogé par des journalistes, l'un d'entre eux répondit ceci à la question de savoir quel article lui plaisait le plus et lequel lui plaisait le moins dans le texte constitutionnel de 1978:

He mirado y remirado la Constitución y no hay un artículo que me guste más ni un artículo que me guste menos. En todos hay de todo. [...] Pero lo que me gustaría más que todo sería [...] dejar la Constitución otra vez tal como está, de forma que a nadie le pueda gustar del todo ni disgustar completamente y pueda ser por eso la Constitución de todos: lo que he llamado la Constitución de la dos Españas ${ }^{21}$. (J'ai regardé et reregardé la Constitution et il n'y a pas un article qui me plaise plus, pas un article qui me plaise moins. Il y a de tout dans tout. [...] Mais ce qui me plairait le plus, [...] ce serait de laisser à nouveau la Constitution telle qu'elle est, de façon à ce qu'elle ne puisse plaire entièrement à personne ni déplaire complètement mais qu'elle puisse être pour cette raison la Constitution de tous : ce que j'ai appelé la Constitution des deux Espagnes.)

Peut-être est-il intéressant de souligner que, sur son lit de mort, le Caudillo s'adressant au futur roi lui rappelle la tâche fondamentale qui lui incombe:

Pero me acuerdo de que [...] me dijo en un suspiro: "Alteza, la única cosa que os pido es que mantegáis la unidad de España $»^{22}$. (Mais je me souviens [...] qu'il m'a dit dans un soupir: «Altesse, la seule chose que je vous demande, c'est que vous sauvegardiez l'unité d'Espagne».)

Étrange écho: de façon tragique, l'allocution prononcée par le roi, le 12 mars 2004 à la suite des sanglants attentats de Madrid (200 morts), renoue avec ce choix initial: unir tous les Espagnols dans une même volonté solidaire. Nommer sert à cela.

20. Voir A. Machado, 1938, Prólogo a los discoursos de Azaña.

21. J. Benet Morell, Sénateur EDC (Entesa des Catalans), cité par J. M. Garcia Escudero et M. .A. Garcia Martinez, 1998, La Constitución dia a dia, los grandes temas de la Constitución de 1978 en el debate parlamentario, Madrid, Congreso de los diputados.

22. J. L. de Vilallonga, 1993, El Rey, conversaciones con D. Juan Carlos I de España, Plaza y Janes, p. 86. 


\title{
Résumé / Abstract / Compendio
}

\author{
Sémantique et Histoire. España et Españas dans le discours \\ de Juan Carlos
}

Les discours annuels du roi d'Espagne Juan Carlos montrent un usage récurrent de la forme España, ce qui n'a rien de surprenant, mais aussi de la forme Españas, ce qui est inattendu. L'opposition de ces deux usages est lourde de sens. Le morphème de nombre, dans ce cas, est chargé de significations que seule l'Histoire peut expliquer.

Mots-clés : nom propre, Espagne, Histoire, discours politique, sémantique.

\section{Semantics and History. España and Españas in Juan Carlos's speeches}

The speeches made every year by the King of Spain Juan Carlos show evidence of the recurrent use of the form España, as can be expected, but also of the unexpected form Españas. The use of these two contrasting forms is quite significant for, in that case, the morpheme " $s$ " is pregnant with meanings that only history can explain.

Key-words : proper name, Spain, History, political discourse, semantics.

Semántica y Historia. España y Españas en los discursos del rey Juan Carlos

Los discursos anuales del rey Don Juan Carlos subrayan un uso recurrente de la forma España, lo que no sorprende, pero tambien aparece la forma Españas, lo que podría estrañar. La oposición en el uso de estas dos palabras es muy significativa. Y en dicho caso, el morfema de número s viene cargado de significaciones especificadas históricamente.

Palabras claves: nombre, España, Historia, discurso politico, semántica. 